\title{
Investigation of wall-slip effect on lead-free solder paste and isotropic conductive adhesives
}

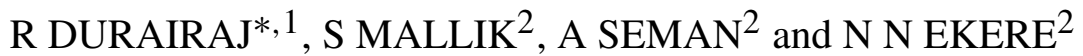 \\ ${ }^{1}$ Faculty of Engineering and Science (FES), Universiti Tunku Abdul Rahman \\ (UTAR), Jalan Genting Kelang, Setapak 53300 Kuala Lumpur, Malaysia \\ ${ }^{2}$ Electronic Manufacturing Engineering Research Group, The Medway School of \\ Engineering, University of Greenwich, Pembroke, Chatham Maritime, \\ Kent ME4 4TB, UK \\ e-mail: rajkumar@utar.edu.my; s.mallik@gre.ac.uk
}

MS received 13 December 2007; revised 19 January 2009

\begin{abstract}
Slippage due to wall depletion effect is well-known in rheological investigation. The aim of this study was to investigate the influence of the paste microstructure on slip formation for the paste materials (lead-free solder paste and isotropic conductive adhesives). The effect of different flow geometries, gap heights and surface roughness on the paste viscosity was investigated. The utilisation of different measuring geometries has not clearly showed the presence of wall-slip in the paste samples. The existence of wall-slip was found to be pronounced when gap heights were varied using the parallel plate geometry. It was also found that altering the surface roughness of the parallel plate measuring geometry did not significantly eliminate wall-slip as expected. But results indicate that the use of a relatively rough surface helps to increase paste adhesion to the plates and to a certain extent inducing structural breakdown in the paste. Most importantly, the study also demonstrated on how the wall-slip formation in the paste material could be utilised for understanding of the paste microstructure and its flow behaviour.
\end{abstract}

Keywords. Wall-slip; solder paste; rheology; isotropic conductive adhesives; stencil printing process.

\section{Introduction}

Wall-slip occurs due to the displacement of the particles away from solid boundaries, leaving a liquid layer, which has apparent lower viscosity than the bulk viscosity (Barnes 1995). The formation of wall-slip is usually seen as a source of error in the rheological measurements and various steps have been taken to eliminate or reduce wall-slip (Kolli et al 1997). However, in some cases the wall-slip is important because most material production involves flow through some form of complex geometry which is surrounded by walls. Hence the formation of slip

${ }^{*}$ For correspondence 


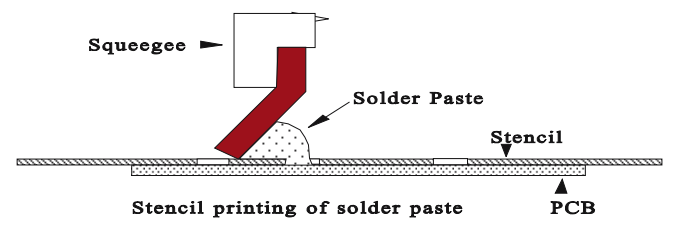

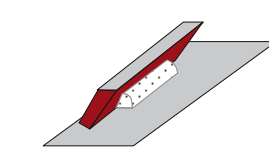

Squeegee Deformation

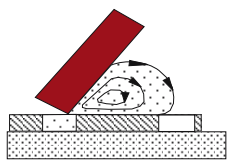

Paste roll

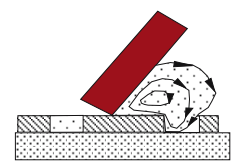

Aperture Filling

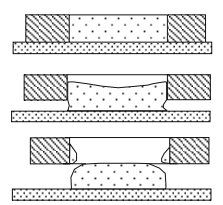

Aperture Emptying

Figure 1. Stencil printing process.

in a material at different stages in a material processing can aid the flowability of the material and could be related to the performance of the material in the real production environment.

In the electronic manufacturing industry, the most popular method to deposit paste material (solder paste and isotropic conductive adhesives) is known as stencil printing, as can be seen in figure 1. During the stencil printing process, the squeegee generates hydrodynamic pressures creating a paste roll and injects the paste into the apertures. In stencil printing process, two critical sub processes are the aperture filling and aperture release process. Successful aperture filling and release will greatly depend on the rheology of the paste material. When the paste is sheared by the squeegee, the viscosity of the paste must be low enough to flow into the aperture. In the aperture release process, the viscosity of the paste must be large enough to overcome the adhesion forces of the sidewall of the apertures. The aperture release process could also be improved with the formation of a lubrication layer between the aperture and stencil wall. A good print is defined by the volume of the paste deposited on the interfaces i.e. the deposited volume is equal to the aperture volume. However, in a manufacturing environment, the volume of deposited is always less than the aperture volume. This may be caused by the adhesion of the paste to the aperture walls. Therefore, it is necessary to engineer a paste that will easily flow through the aperture and able to achieve consistent printing, which will reduce defects and increase production yield.

The objective of the study is to investigate the influence of paste microstructure on wall-slip formation. The experimental work performed in this study was focused on three main areas namely; (i) the effect of different geometries, (ii) gap height and (iii) the surface roughness on the paste viscosity measurements.

\section{Experimentation}

\subsection{Instrumentation and sample preparation}

The rheological measurements were performed using a Reologica StressTech controlled stress rheometer (Rheologica Instruments AB, Sweden). Two solder pastes and one isotropic conductive adhesive, ICA (named as P1, P2 and P3) were investigated in this study. The details of these paste materials are given in table 1 . The samples were taken out of the refrigerator prior to rheological measurements and were left for at least 4 hours to allow the paste to 
Table 1. Constituent of solder paste and ICA's investigated.

\begin{tabular}{lccll}
\hline Sample & $\begin{array}{c}\text { Particle size } \\
\text { distribution }(\mu \mathrm{m})\end{array}$ & Particle shape & \multicolumn{1}{c}{ Composition } & Filler material \\
\hline P1 & $5-15$ & Spherical & Tin/Silver/Copper & No clean Flux \\
P2 & $5-10$ & Spherical & Tin/Silver/Copper & No clean Flux \\
P3 & $5-8$ & Flakes & Silver & Epoxy Resin \\
\hline
\end{tabular}

achieve room temperature. To restore samples to their specified rheology, the samples were then stirred gently for 15-30 seconds before being applied on to the rheometer. After the sample was loaded on to the rheometer a further rest period of about 1 minute was followed before commencing the test. Identical loading procedures were followed in all the tests. All the rheological measurements were carried out at $25^{\circ} \mathrm{C}$ (accuracy $\pm 0 \cdot 1{ }^{\circ} \mathrm{C}$ ), where each test was repeated three times (with fresh samples used for each test).

\subsection{Methodology}

The shear stress sweep test was used to study the effect of plate geometry, gap height and plate surface roughness on paste viscosity. In the shear stress sweep test, the viscosity $(\eta)$ is measured as a function of applied stress (Pa). The paste samples were sheared from 100$1000 \mathrm{~Pa}$. The summary of the experimental conditions used in the study are presented in table 2.

\subsection{Measurement of the surface roughness of the parallel plate geometries}

The surface roughness of the parallel plates used in this study was measured using the Zygo NewView 5000. In the study, three different plate materials were utilised namely; stainless steel plate, aluminium grooved plate and plastic plate. The surface roughness was measured across a distance of $40 \mathrm{~mm}$, which is also the diameter of the parallel plates. The measurement was repeated three times and the average surface roughness $\left(R_{a}\right)$ was recorded for different plate material is presented in table 3 .

\section{Analysis and discussion of results}

\subsection{Effect of plate geometry on viscosity measurements}

The results of the effect of using two different plate geometries, namely, the cone and plate, and the parallel plate is presented in this section. In this study, a cone and plate of $40 \mathrm{~mm}$

Table 2. Summary of experimental test parameters.

\begin{tabular}{|c|c|c|}
\hline Experimental focus & Gap height & Types of geometry \\
\hline Effect of plate geometry on viscosity & $1.0 \mathrm{~mm}$ & Parallel plate and cone and plate \\
\hline Effect of gap height on viscosity & $1 \mathrm{~mm}, 2 \mathrm{~mm}$ and $3 \mathrm{~mm}$ & Parallel plate \\
\hline $\begin{array}{l}\text { Effect of surface roughness on } \\
\text { viscosity }\end{array}$ & $1.0 \mathrm{~mm}$ & $\begin{array}{l}\text { Parallel plate with different } \\
\text { surface roughness }\end{array}$ \\
\hline
\end{tabular}


Table 3. Results of the measurement of the surface roughness.

\begin{tabular}{lc}
\hline Parallel plate material & Surface roughness, $R_{a}(\mu \mathrm{m})$ \\
\hline Stainless steel & 0.00173 \\
Plastic & 0.132 \\
Aluminium (grooved) & 2.64 \\
\hline
\end{tabular}

diameter and cone angle of $4^{\circ}$, and a parallel plate of $40 \mathrm{~mm}$ diameter with a gap height of $1.0 \mathrm{~mm}$ was used. Figure 2 shows a plot of the viscosity as a function of shear stress for the $\mathrm{P} 1, \mathrm{P} 2$ and $\mathrm{P} 3$ samples.

The viscosity measured with the parallel plate and cone plate geometries were similar for the $\mathrm{P} 1$ and $\mathrm{P} 2$ pastes. For the $\mathrm{P} 1$ pastes, the viscosity drops as the shear stress increases; this indicates a shear thinning behaviour in the paste. As expected, the measured viscosity for the $\mathrm{P} 2$ paste was higher than the $\mathrm{P} 1$ paste, which could be due to the smaller particle sizes in the $\mathrm{P} 2$ paste, resulting in a stronger interactive forces between flux and solder alloy powder particle. However, a closer observation of the viscosity plot for the P2 paste showed an increase in viscosity between 100 and $150 \mathrm{~Pa}$ and beyond this stress value the viscosity decreases. As stated earlier, the initial increase in viscosity observed for the P2 paste could be attributed to the interaction between the flux medium and the solder powder particles. As a result, the applied shear stress is not strong enough to overcome the interactive forces between the solder powder particles and flux medium. The finding from this study is in good agreement with a previous viscosity studies conducted on the P1 and P2 paste (Durairaj et al 2004).

In contrast, the viscosity measured for $\mathrm{P} 3$ was lower than the $\mathrm{P} 1$ and $\mathrm{P} 2$ pastes. As expected, the viscosity of the P3 decreases with increasing shear stress. These results are in line to the reported results of Kay et al (2003) and Durairaj et al (2004). However, a large difference was observed in the viscosity measured for the P3 paste for the parallel plate geometries

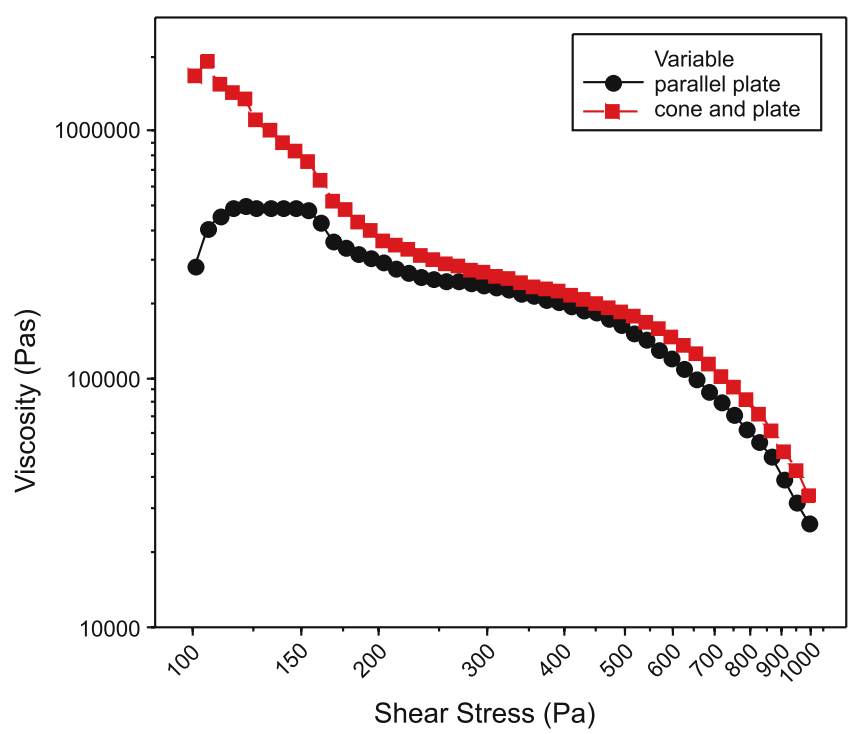

(a)
Figure 2. (Continued). 


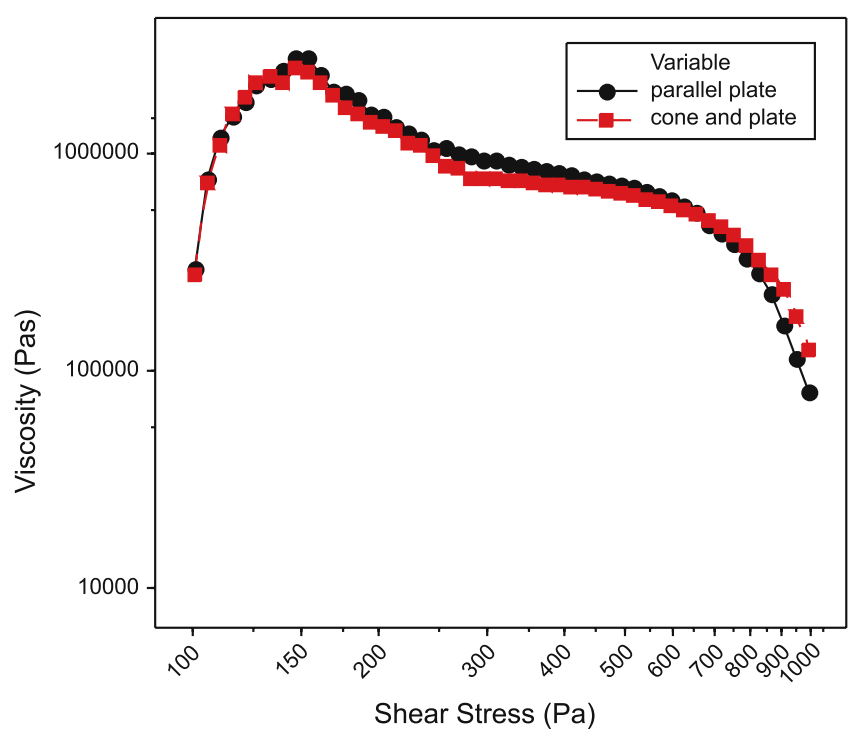

(b)

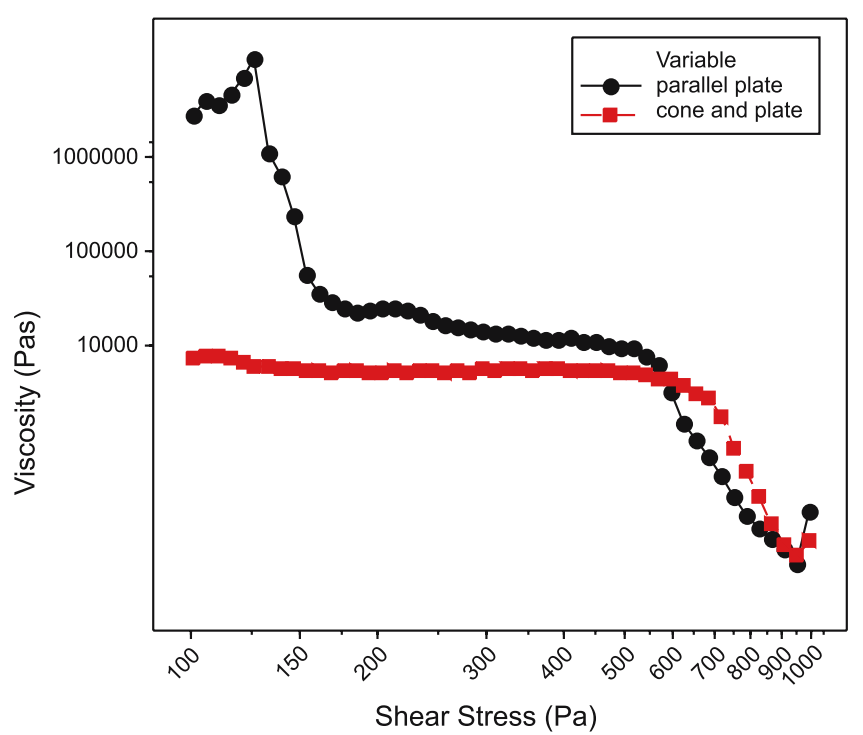

(c)
Figure 2. Effect of geometry on viscosity for sample (a) P1, (b) P2 and (c) P3.

when compared to the cone and plate geometry. The viscosity measured using the cone and plate geometry did not show a significant change until it reached a shear stress value of $720 \mathrm{~Pa}$ and beyond this stress value the viscosity decreased drastically. The almost constant viscosity measured up to $720 \mathrm{~Pa}$ is not possible due the fact that the P3 is not a Newtonian material and the constant viscosity could be due to the generation of a liquid-rich layer at the interface between the sample and the measuring geometry causing a lower viscosity reading. 
Another possible reason for the constant viscosity for the P3 sample is the possible development of secondary flow, which could be attributed to the jamming of the silver flakes at adjacent to the tip of the cone and plate. As a result, the generation of the secondary flow within the cone and plate could cause the separation between the silver flakes and polymer binder sample to crawl out of the instrument under the influence of centrifugal forces and elastic instabilities. This can then lead to higher volume of sample distribution at the edgeresulting in inaccuracies in the measurement. The drop in viscosity after $720 \mathrm{~Pa}$ for the cone and plate geometry could possibly be due to $\mathrm{P} 3$ pastes re-establishing contact with the cone and plate geometry.

Based on the results obtained in this study of three different pastes, we can see that the viscosity measurements are largely independent of the plate geometry used between the $150 \mathrm{~Pa}$ to $600 \mathrm{~Pa}$ shear stress range. Further experiments are of course required to determine the source of the minor variation observed in the measurements in this range. The P3 samples seem to respond well when tested under parallel plate geometry compared to the cone and plate geometry. However, care should be taken to ensure that the viscosity data obtained are not influenced by wall-slip formation. This can be achieved by repeating the test (at least 3 times) and comparing the viscosity data with other sources for example paste manufacturers data sheet or measurement carried out on another rheometer.

\subsection{Effect of gap height of parallel plate geometry on the viscosity measurements}

The result of the effect of gap height on the viscosity measurement is presented in this section. It should be noted that in this study the gap height is the distance between the top plate (rotating geometry) and the bottom plate (usually stationary) and the gap heights used were $1 \mathrm{~mm}, 2 \mathrm{~mm}$ and $3 \mathrm{~mm}$ respectively. A plot of the viscosity as a function of shear stress for these three gap heights is shown in figure 3 .

The results show that the viscosity measurements are affected by changing of gap heights for the parallel plate geometry. However, it is very difficult to identify a definite trend in the measurements in terms of the exact effect of gap height on the viscosity measurements.

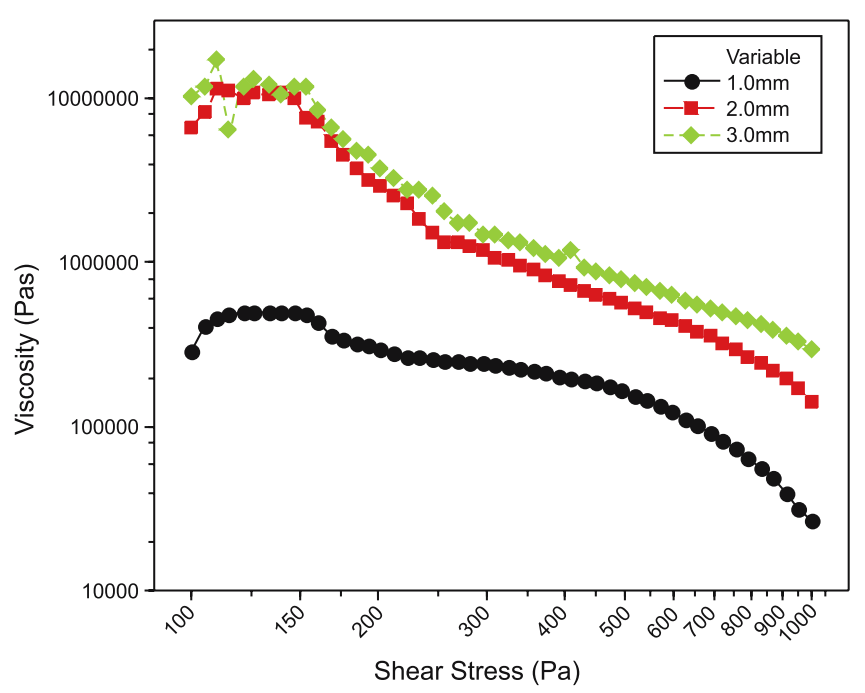

(a) P1
Figure 3. (Continued). 


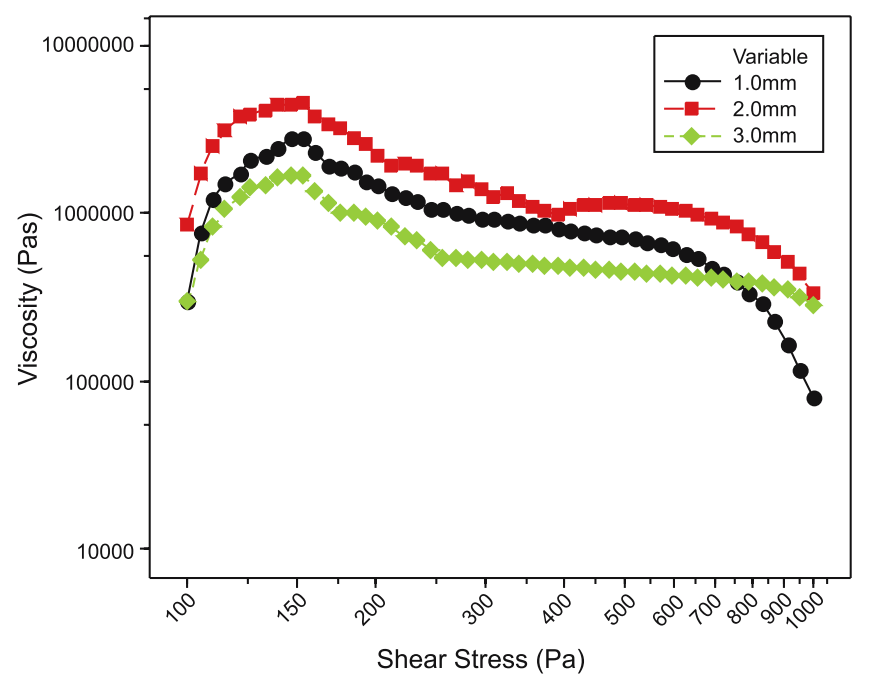

(b) P2

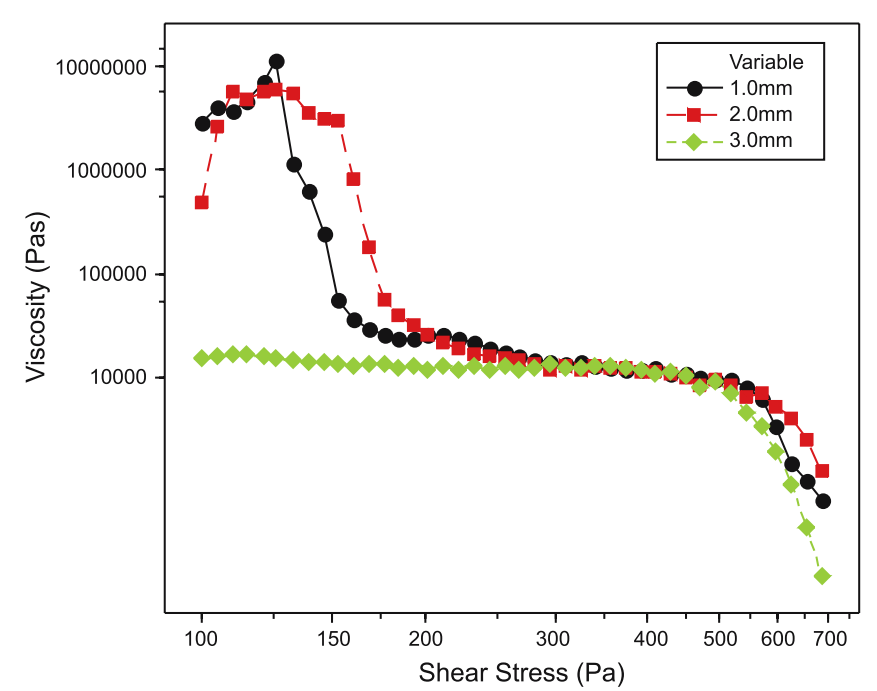

(c) P3
Figure 3. Effect of gap height on viscosity data for (a) P1, (b) P2 and (c) P3 paste samples.

The mathematical equations derived to calculate the viscosity of materials does not account for wall-slip and non-laminar flow during rheological measurements. Hence, the variation in the viscosity data may well be due to formula incorporated within the rheological software, which assumes that there is no slip formation and the flow within the measuring plates are laminar. According to Whorlow (1980) the most obvious manifestation of slip is the one obtains different results for different size geometries (e.g. gap size, tube radius, etc) because the formulas used to calculate viscosities assumes no slip in the flowing liquid. In particular the apparent viscosity calculated in this way always decreases with decrease in geometry size e.g. tube radius, gap size, cone and plate.

For the P1 pastes, the viscosity increases with increasing gap heights and the difference in the viscosity measured at different gap heights could indicate the presence of wall-slip in 
the parallel plate. The result obtained in this study is similar to those reported in the previous study by Kaylon et al (1993) and Riedlin (1998), who also found the viscosity values increase with increasing gap height. The effect of the gap height on viscosity measurements could be due to the sensitivity to the surface area to volume ratio of the plate geometry being utilised. However, both these authors did not provide any satisfactory information on the difference in the viscosity results with different gap height. One possible explanation is that, as the plates are rotating, the centrifugal forces pushes the sample towards the edge of the measuring plates and as the gap height is increased, the volume of paste within the plates will also, which could lead to an increase in the volume of the sample at the edge measuring plates. As stated previously, that the shear stress and shear rate is dependent on the radius of the plate. Most rheometers calculate the shear stress and shear rate at the edge of the plate as the reference point to determine the shear rate and shear stress. Based on the way the rhoemeter calculate the shear stress and shear rate, any changes in the volume of the paste at the edge of interface of the parallel plate could have an affect on the viscosity data.

In contrast, for the $\mathrm{P} 2$ pastes, the effect of the gap on the viscosity followed a similar trend to $\mathrm{P} 1$ for gap $1.0 \mathrm{~mm}$ and $2.0 \mathrm{~mm}$. But at the gap $3.0 \mathrm{~mm}$, the viscosity measured was lower than that of the viscosity measured at gap $1.0 \mathrm{~mm}$. The large drop in viscosity measured for the P2 paste at the gap of $3 \mathrm{~mm}$ seem to suggest that the smaller solder alloy powder particles size in P2 pastes will have larger surface area resulting in a stronger interaction between the particles and the flux medium. Barnes (2000) suggested that small particles could result in large colloidal effects, and hence flocculation of the particles. If the system of particles is flocculated, then even small particles can give rise to a large effective particle size and gravitational effect could set in causing the particle to separate from the medium. For these reasons greater care should to be taken with when testing smaller particles systems with small gap heights. It is possible to attribute the effect for two reasons, first, the effective size of flocculates can be as big as $100 \mathrm{~s}$ of microns, and second, the effective phase volume is much bigger than real value, thus exacerbating the wall-slip formation. Furthermore, as the shear stress increases beyond $700 \mathrm{~Pa}$, a large drop in viscosity was measured at the gap of $1.0 \mathrm{~mm}$, which could indicate structural breakdown of the P2 paste due to increasing shear stress. For the $\mathrm{P} 3$ paste, the viscosity measured at gap $2 \mathrm{~mm}$ was similar to that measured at gap $1 \mathrm{~mm}$, whereas the viscosity measured at $3 \mathrm{~mm}$ was considerably lower. The viscosity measured at the $3 \mathrm{~mm}$ gap height could be affected by the wall-slip because it is unusual for the pastes' viscosity to remain constant with increasing shear stress. One possible explanation for this is that due to polymer chain adsorption, the polymer materials tend to adhere better to metallic surface such as steel or aluminium (Wang 1999). In the case of P3, the polymer chains could have been sliding at the interface between the parallel plate and the sample causing a low viscosity measurement.

The difference in viscosity data for different gap height for each of the paste would indicate that wall-slip formation in each paste could be due to the paste formulation. Hence the slip formation in the paste could be used as unique characteristic of the pastes. Previous studies have also observed similar variation in the viscosity data as a result of changes in the gap height between the top plate and bottom plates (Burton et al 1983, Yoshimura et al 1988, Kaylon et al 1993, Kaylon 1995 and Riedlin 1998).

\subsection{Effect of surface roughness of parallel plate geometry on the viscosity measurements}

This section presents the result of the effect of surface roughness of the parallel plates on the viscosity measurement for a constant gap height of $1.0 \mathrm{~mm}$. A plot of the effect viscosity versus shear stress for surface roughness of the parallel plates is shown in figure 4 . The details 
of the surface roughness value $\left(\mathrm{R}_{\mathrm{a}}\right)$ of the plate geometry used in this study can be seen in table 3 . The viscosity plots for the P1 pastes, indicate that the two parallel plates with the $\mathrm{R}_{\mathrm{a}}$ value of $0.00173 \mu \mathrm{m}$ and $0.132 \mu \mathrm{m}$ showed similar viscosity readings, whereas the parallel plate with the highest $R_{a}$ value (roughest plate) produced the highest viscosity data. The increase in the viscosity could be due to the increased adhesion of the P1 paste to the measuring plate surface, which could have reduced the stick/slip effect resulting in a high viscosity reading.

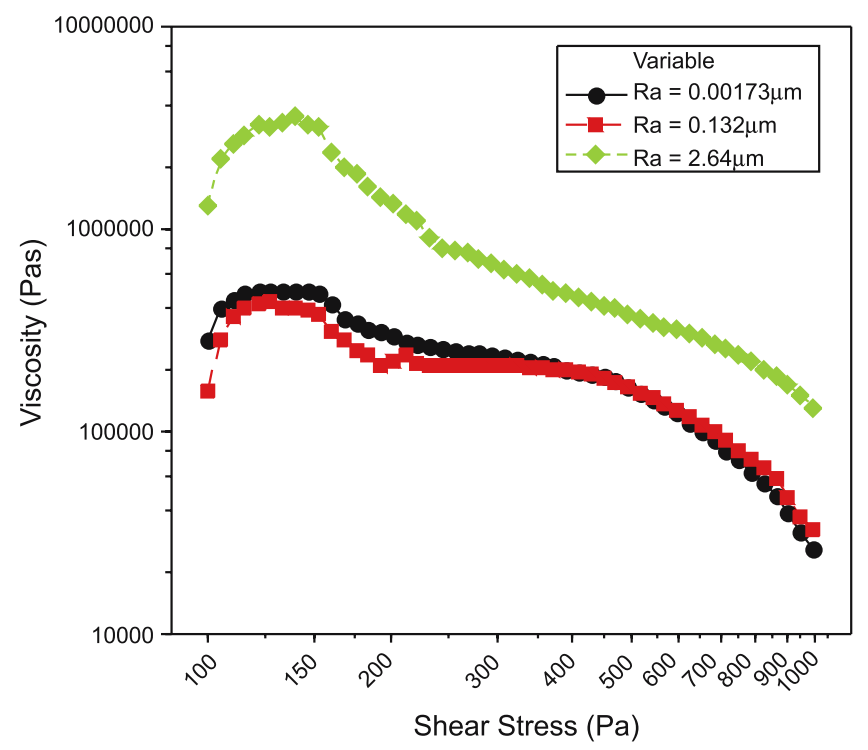

(a)

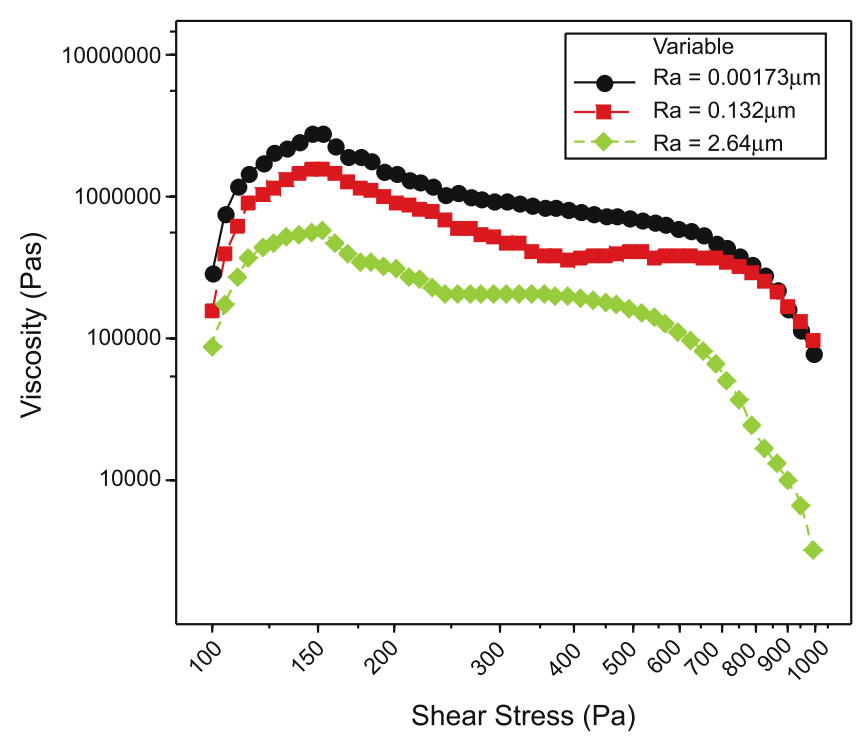

(b)

Figure 4. (Continued). 


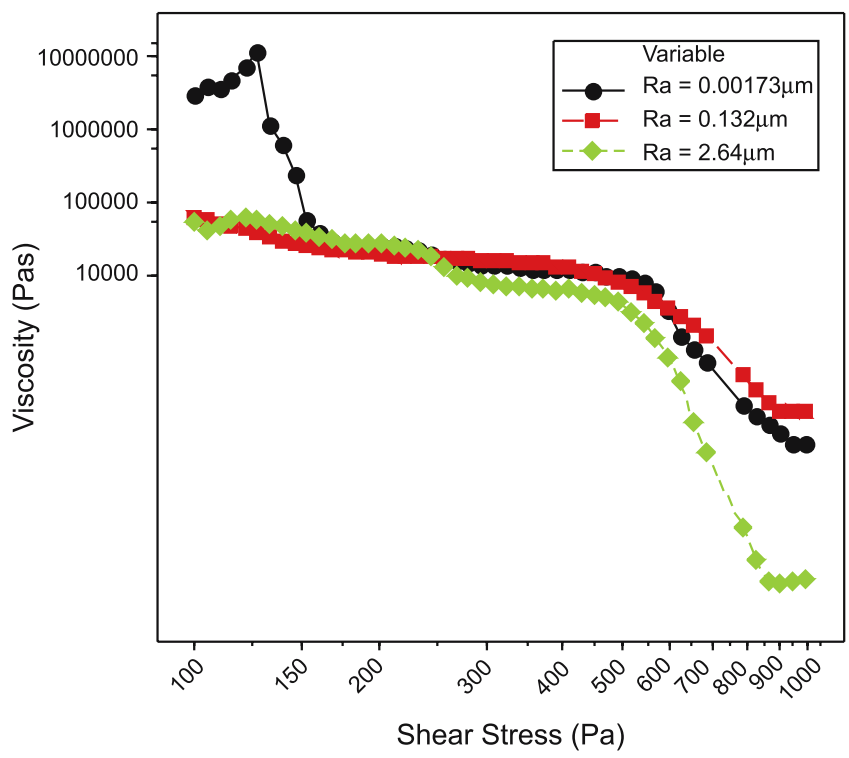

(c)
Figure 4. Effect of surface roughness on the viscosity for (a) P1, (b) P2 and (c) P3 paste samples.

For the P2 pastes, the increase in the surface roughness of the parallel plate geometry caused a drop in viscosity. The drop in viscosity could have been due to the sample fracturing either at the interface or at the bulk material. A previous study by Kolli et al (1997) on solder pastes, observed internal slip in the bulk of the solder paste sample about midway between the gap heights, ultimately leading to the expulsion of part of the sample. The author also found that the time at which this internal slip occurred was approximately ten seconds after the test was underway and thereafter, shear fracture was observed to take place in the samples. In an another study by Kaylon et al (1994) on the effect of surface roughness of dense suspensions found that parallel plate with higher surface roughness were sometimes effective in preventing wall-slip, but when the parallel plate with $R_{a}$ values exceeding $8.0 \times 10^{-3}$ were used, sample fracture was observed for all shear rates. In the case of P2 pastes, the largest $R_{a}$ value of $2.64 \mu \mathrm{m}$ used in this study clearly exceed the value utilised by Kaylon (1995). As a result after $600 \mathrm{~Pa}$, a large drop in viscosity was observed, which could indicate that the shear fracture could have occurred in the P2 samples. In addition, the tips of the asperities of the roughened surfaces might act as stress concentration points at the disk walls, initiating the fracture phenomena.

In the P3 pastes, a similar viscosity was measured for the parallel plate with $\mathrm{R}_{\mathrm{a}}$ value of $0.00173 \mu \mathrm{m}, 0.132 \mu \mathrm{m}$ and $2.64 \mu \mathrm{m}$ up to the applied shear stress of $500 \mathrm{~Pa}$. It is known that the adhesion at metal interface with polymeric material is influenced by both the roughness and the material of the measuring plate (Wang 1999). Thus, it is possible for the P3 sample to be affected in a similar manner suggested by (Wang 1999). However, further investigation is required to gain an understanding of the interaction between the P3 samples with the parallel plate material.

The study by Chen et al (1994) on polymeric materials showed that the lowest adhesion to the stainless steel plate and the highest adhesion is to the glass plate. The author also concluded that for polymeric materials, the wall-slip formation is influence by the surface roughness and material of the measuring geometry. The study on the P3 paste seems to suggest that 
for the plate with the lowest surface roughness (stainless steel), a sudden drop in viscosity was observed from 100 to $150 \mathrm{~Pa}$, which could be due to the formation of slip layer. As the shear stress increases, the similarities in the viscosity data up to $500 \mathrm{~Pa}$ could indicate that the wall-slip formation is minimised due to adhesion characteristic of the P3 material to different parallel plate geometries. However, further work may be necessary to study the adhesion characteristic of P3 with different plate materials. But beyond $500 \mathrm{~Pa}$ stress value, all the paste showed a drop in viscosity indicating structural breakdown in the samples. But for the sample sheared using the measuring geometry with $\mathrm{R}_{\mathrm{a}}$ value of $2.64 \mu \mathrm{m}$ showed a large drop in viscosity. As stated previously, the use of a rougher surface may not necessarily help in preventing wall-slip formation but could contribute to the structural breakdown in the pastes.

\section{Conclusions}

The study on the effect of the plate geometry namely; parallel plate and cone and plate geometry seem to suggest that viscosity measured using the cone and plate could have been affected by slip formation at the interface of the plate surface and the top of the sample. From the study, the results were inconclusive and for the plate geometry utilised, changing the plate geometry did not clearly show the presence of wall-slip in the pastes samples.

The study to investigate the effect of gap height on the viscosity measurements revealed that the variation in the measured viscosity with increase in gap heights may indicate the effect of wall-slip formation when using the parallel plate measuring geometry. Finally, the study of the effect of surface roughness on the viscosity measurement was carried out by using three parallel plates with different surface roughness. The expectation was that increasing the surface roughness of the plate surface will help to reduce the wall-slip, and thus reduce the variation in measured viscosity. In general, the results showed that altering the surface roughness did not significantly reduce the wall-slip effect - but rather may have helped to induce structural breakdown of the paste material (as was observed with the P2 pastes in particular).

The authors would like to acknowledge the Engineering and Physical Sciences Research Council (EPSRC), UK and Henkel Loctite Multicore, UK for funding this work.

\section{References}

Barnes H A 2000 A handbook of elementary rheology, Insitute of Non-Newtonian Fluid Mechanics, University of Wales.

Barnes H A 1995 A review of the slip (wall depletion) of polymer solutions, emulsions and particle suspensions in viscometer: its causes, character, and cure. J. Non-Newtonian Fluid Mechanic 56: 221-251

Burton R H, Folkes M J, Narh K A, Keller A 1983 Spatial variation in viscosity in sheared polymer melts. J. Mater. Sci. 18(1): 315-320

Chen Y L, Larson R G, Patel S S 1994 Shear fracture of polystyrene melts and solutions. Rheologica Acta 33(4): 243-256

Durairaj R, Ekere N N, Salam B 2004 Thixotropy flow behaviour of solder and conductive adhesives paste. J. Material Science: Materials in Electronic 15: 677-683

Kaylon D M, Yaras P, Yilmazer U 1993 Rheological behaviour of a concentrated suspension: A solid rocket fuel simulant. J. Rheology 37/1: 35-53 
Kaylon D M 1995 Characterizing high-solid suspensions. Chem. Tech. 25(5): 22-30

Kay R W, Desmulliez M P, Bailey C, Stoyanov S, Glinski G, Ekere N N, Durairaj R, Hendriksen M, Smith B, Ongley P, Price D, Roberts A, Whitmore M, Gourley J 2003 A high volume, low cost and low temperature MEMS packaging technology based on a flip-chip assembly process, VDE Microtec Conference.

Kolli V G, Gadala-Maria F, Anderson R 1997 Rheological characterisation of solder paste for surface mount applications. IEEE Transactions on Components, Packing and Manufacturing Technology, Part B 20(4): 416-423

Riedlin M H A 1998 Rheological characterisation of solder paste used in the stencil printing process, $\mathrm{PhD}$ Thesis, University of Salford.

Whorlow R W 1980 Rheological Techniques, Horwood Ltd., Chichester

Wang S H 1999 Molecular transitions and dynamics at polymer/wall interfaces: Origins of flow instabilities and wall-slip. Advances in Polymer Science 138: 229-275

Yoshimura A, Prud'homme R K 1988 Wall-slip corrections for coquette and parallel disk viscometer. J. Rheology 32/1: 53-67 